\title{
DISCRETE FRACTIONAL HILBERT TRANSFORM
}

\author{
Soo-Chang Pei ${ }^{1}$ and Min-Hung Yeh $^{2}$ \\ ${ }^{1}$ Department of Electrical Engineering, National Taiwan University, Taipei, Taiwan, R. O. C. \\ ${ }^{2}$ Department of Computer Information Science, Tamsui Oxford University College, Tamsui, Taipei, Taiwan, R. O. C.
}

\section{ABSTRACT}

Hilbert transform plays an important role in the signal processing. A generalization of Hilbert transform, fractional Hilbert transform, was recently proposed, and it presents physical interpretation in the definition. In this paper, we develop the discrete fractional Hilbert transform, and apply the proposed discrete fractional Hilbert transform to the edge detection applications.

\section{INTRODUCTION}

Hilbert transform plays an important tool for signal processing, and it has been used in many areas, such as modulation theory[1], edge detection [2] and so on. For instance, in communication the Hilbert transform is commonly used for single side-band modulation. This modulation can save band width for transmission. Besides the continuous Hilbert transform, the discrete Hilbert transform, can also be used for digital communication and edge detection of digital image[1] [2].

A generalization of Hilbert transform, fractional Hilbert transform, was proposed in [3]. The fractional Hilbert transform can process signal in the fractional Fourier plane instead of conventional Fourier plane. The method for implementing fractional Hilbert transform in [3] is using optical instruments. It is still lack of digital implementation for fractional Hilbert transform computation. The goal of this paper is to develop the discrete fractional Hilbert transform, which can have similar outputs as those of continuous fractional Hilbert transform. Moreover, we will apply the proposed discrete fractional Hilbert transform to the edge detection applications.

\section{HILBERT TRANSFORM}

\subsection{Continuous Hilbert Transform}

The Hilbert transform of a continuous signal $x(t)$ is computed as:

$$
\hat{x}(t)=\int_{-\infty}^{\infty} \frac{x(\tau)}{t-\tau} d \tau
$$

The continuous Hilbert transform consists of a $\frac{\pi}{2}$ radian phase shift (for positive frequencies only) in the frequency domain[4]. Thus the transfer function of Hilbert transform becomes:

$$
H_{1}(\omega)= \begin{cases}j & \omega>0 \\ 0 & \omega=0 \\ -j & \omega<0\end{cases}
$$

\subsection{Discrete Hilbert Transform}

The transfer function of the discrete Hilbert transform is defined as:[4]

$$
H(\omega)= \begin{cases}j & 0<\omega<\pi \\ 0 & \omega=0 \text { and } w=\pi \\ -j & -\pi<\omega<0\end{cases}
$$

The discrete Hilbert transform can be computed through the following steps:[4]

- Step1: Compute the DFT of signal $\{\mathbf{x}[k]\}$.

$$
\mathbf{X}[n]=D F T[\mathbf{x}[k]]
$$

- Step2: $\mathbf{X}[n]$ is multiplied by the mask $\mathbf{M}_{\mathbf{1}}$. The mask $\mathbf{M}_{\mathbf{1}}$ is defined as:

If $N$ is even,

$$
\mathbf{M}_{\mathrm{I}}=[0, \underbrace{j, j, \cdots, j}_{\frac{N}{2}-1}, 0, \underbrace{-j,-j, \cdots,-j}_{\frac{N}{2}-1}]
$$

If $N$ is odd,

$$
\mathbf{M}_{1}=[0, \underbrace{j, j, \cdots, j}_{\frac{N-1}{2}}, \underbrace{-j,-j, \cdots,-j}_{\frac{N-1}{2}}]
$$

- Step3: Compute the inverse DFT to obtain $\hat{\mathbf{x}}[k]$.

$$
\hat{\mathbf{x}}[k]=I D F T\left[\mathbf{X}[n] \mathbf{M}_{1}[n]\right]
$$

Then $\hat{\mathbf{x}}[k]$ will be the discrete Hilbert transform of $\mathbf{x}[k]$.

The block diagram for implementing discrete Hilbert transform is drawn in Fig.2.

\section{DEVELOPMENT OF DISCRETE FRACTIONAL HILBERT TRANSFORM}

In [3], two alternative definitions for the continuous fractional Hilbert transform have been developed. One is based upon the modification of spatial filter with a fractional parameter, and its transfer function is defined as:

$$
H_{P}(v)=\cos \phi H_{0}(v)+\sin \phi H_{1}(v)
$$

where $\phi=\frac{P \pi}{2}$. The definition of fractional Hilbert transform in (8) is a weighted sum of the original signal and its conventional Hilbert transform. 
The other fractional Hilbert transform is based on the fractional Fourier transform(FRFT) that was introduced recently in [5]. The FRFT operation indicates a rotation of signal in the time-frequency plane. When $\alpha=0$, the FRFT is an identity transform. While $\alpha=\frac{\pi}{2}$, the FRFT will become conventional Fourier transform.

The transfer function for the other fractional Hilbert transform based upon the FRFT method is defined as:

$$
V_{Q}=\mathcal{F}^{-Q} H_{1} \mathcal{F}^{Q}
$$

where $\mathcal{F}^{Q}$ is the fractional Fourier transform with fractional order $Q$. While $Q=1$, the FRFT becomes the conventional Fourier transform. $Q=\alpha \frac{2}{\pi}$.

The block diagrams for implementing fractional Hilbert transform are drawn in Fig.1. Fig.1(a) shows the block diagram for the spatial filter with fractional parameter $P$. Fig.1(b) shows the block diagram for the FRFT method in fractional Hilbert transform computation. Both fractional Hilbert transform approaches were invented according to the postulate: the first-order fractional Hilbert transform should be consistent with the conventional Hilbert transform.

Moreover, the above two definitions can be merged into a general definition of fractional Hilbert transform. Thus its transfer function is defined as:

$$
\mathcal{H}_{P, Q}=\mathcal{F}^{-Q} H_{P} \mathcal{F}^{Q}
$$

The combination of the two fractional schemes is shown in the Fig.1(c).

In the other way, the discrete fractional Hilbert transform can also be defined in two ways. One is based upon the modification of spatial filter with a fractional parameter; the other is based on the discrete fractional Fourier transform(DFRFT). The methods for computing DFRFT were recently proposed in [6] and [7]. Because the method in [6] cannot provide the similar results as those of continuous case, we adopt the DFRFT computation defined in [7]. Thus the kernel of DFRFT is defined as:

$$
\mathbf{F}^{Q}=\sum_{n} e^{-j n Q \frac{\pi}{2}} \mathbf{v}_{n} \mathbf{v}_{n}^{*}
$$

where $\mathbf{v}_{n}$ is the $n$-th order DFT Hermite eigenvector. While $Q=1$, the DFRFT will become conventional DFT. Similar to the continuous case, the two definitions of discrete fractional Hilbert transform can also be merged into one. Thus the discrete fractional Hilbert transform can be computed through the following steps:

- Step1: Compute the DFRFT of signal $\{\mathbf{x}[k]\}$ with parameter $Q$.

$$
\mathbf{X}_{Q}[n]=D F R F T_{Q}[\mathbf{x}[k]]
$$

- Step2: $\mathbf{X}_{Q}$ is multiplied by the mask $\mathbf{M}_{P}$. The mask $\mathbf{M}_{P}$ is defined as:

If $N$ is even,

$$
\mathbf{M}_{\boldsymbol{P}}=[\cos \alpha, \underbrace{e^{j \alpha}, e^{j \alpha}, \cdots, e^{j \alpha}}_{\frac{N}{2}-1}, \cos \alpha, \underbrace{e^{-j \alpha}, e^{-j \alpha}, \cdots, e^{-j \alpha}}_{\frac{N}{2}-1}]
$$

If $N$ is odd,

$$
\mathbf{M}_{P}=[\cos \alpha, \underbrace{e^{j \alpha}, e^{j \alpha}, \cdots, e^{j \alpha}}_{\frac{N-1}{2}}, \underbrace{e^{-j \alpha}, e^{-j \alpha}, \cdots, e^{-j \alpha}}_{\frac{N-1}{2}}]
$$

where $\alpha=P \frac{\pi}{2}$.

- Step3: Compute the DFRFT with parameter $-Q$.

$$
\hat{\mathbf{x}}[k]=D F R F T_{-Q}\left[\mathbf{X}_{Q}[n] \mathbf{M}_{P}[n]\right]
$$

Then $\hat{\mathbf{x}}[k]$ is the discrete fractional Hilbert transform of $\mathbf{x}[k]$.

The responses in the fractional Fourier domain are defined $e^{j \alpha}$ and $e^{-j \alpha}$ for positive and negative transform domains, respectively. The first and central entries in the mask $M_{P}$ are both equal to $\cos \alpha$, which are defined as the midpoint of the responses for positive and negative transform domains.

$$
\cos \alpha=\frac{e^{j \alpha}+e^{-j \alpha}}{2}
$$

Then it can be easily verified that (5) (6) are just special cases of (13) and (14). So the proposed discrete fractional Hilbert transform is a generalized version of the conventional discrete Hilbert tranisform. While $P=0$, the mask becomes:

$$
\mathbf{M}_{\mathbf{0}}=[1,1,1, \cdots, 1]
$$

The output of discrete fractional Hilbert transform is the same as the input signal. In this case, the discrete fractional Hilbert transform will become an identity transform. While $P=2$, the mask becomes:

$$
\mathbf{M}_{2}=[-1,-1,-1, \cdots,-1]
$$

The output of discrete fractional Hilbert transform is the negative value of the input signal.

The block diagram shown in Fig. 2 can also be modified for the implementation of discrete fractional Hilbert transform. The DFT and IDFT' must be changed into DFRFT with parameter $Q$ and $-Q$, respectively. And the mask $M_{P}$ in (13) and (14) must be used for the mask block in Fig.2. Example 1:

The amplitudes of discrete fractional Hilbert transform for a rectangular window are shown in Fig. 3 and 4 . It can be observed that the discrete fractional Hilbert transform of a rectangular function consists of two which mark the edges in the signal. While $0<P<1$, the positive edges are emphasized. And the negative edges will be emphasized when $1<P<2$. But in the case of $Q=0.5$, there is no preference either for the negative or for the positive derivative.

The results in Example 1 are very similar to those of the continuous fractional Hilbert transform for the continuous rectangular window shown in [3]. This can help us to verify that the proposed discrete fractional Hilbert transform is our desired transform.

Example 2:

In this example, we will define and compute the discrete fractional analytic signal for a sinusoids signals. The transfer function of fractional analytic signal is defined as:

$$
A_{P}(v)=\cos \phi H_{0}(v)+j \sin \phi H_{1}(v)
$$


where $\phi=P \frac{\pi}{2}$. While $P=0.5$, the output is the traditional analytic signal. The definition in eq. (19) are similar to that of eq. (8). Only the imaginary opertation are substituted for the conventional Hilbert transform part. The spectrum of the output signals for sinusoids signals are drawn in Fig.5. It can be viewed that the outputs are a weighted summation of spectrum of signal and its analytic signal

\section{EDGE DETECTION BY DISCRETE FRACTIONAL HILBERT TRANSFORM}

The conventional discrete Hilbert transform has been applied to find out the edges of digital images [2]. In Example 3 and Example 4, we will apply the discrete fractional Hilbert transform to detect the edges for digital images. The principle used in the following examples for edge detection through discrete fractional Hilbert transform is based up on the idea in The edges occur in the $(m, n)$ point if the following equation is satisfied.

$$
\left|h_{P_{x}, Q_{x}}(m, n)\right|^{2}+\left|h_{P_{y}, Q_{y}}(m, n)\right|^{2}>\text { Threshold }
$$

where $h_{P_{x}, Q_{x}}(m, n)$ is the output of the discrete fractional Hilbert transform with parameters $P_{x}$ and $Q_{x}$ in the $x$ direction for point $(m, n) . h_{P_{y}, Q_{y}}(m, n)$ is the output of the discrete fractional Hilbert transform with parameters $P_{y}$ and $Q_{y}$ in the $y$-direction for point $(m, n)$. The selection of threshold value is to control the amount of edges in detection. The selections of parameters $\left(P_{x}, P_{x}, Q_{x}, Q_{y}\right)$ are depended upon the desired edges in images. $P_{x}=0$ indicates no detection in the $x$-direction; $0<P_{x}<1$ emphases the positive edges in the $x$-direction. $P_{x}=1$ indicates the edge detection in the $x$-direction no matter of positive or negative edges. $1<P_{x}<2$ emphases the negative edge in the $x$-direction. These cases are also the same for the $P_{y}$ parameter in the $y$-direction.

Example 3:

In this example, we will apply the discrete fractional Hilbert transform for the edge detection of digital image. The original image is drawn in the upper left corner of Fig.6, and it is a simple square. The other fifteen images are the detection results. It can be observed that the edges in digital images can be detected through the choices of parameter $P_{x}$ and $P_{y}$. The parameters $Q_{x}$ and $Q_{y}$ used in this example are both equal to 1 . While $P_{x}=0.5$ and $P_{y}=$ 0 , only the horizontal positive edges are emphasized. When $P_{x}=1.5$ and $P_{y}=0$, only the horizontal negative edges are emphasized. These results can be viewed clearly in Fig.6. The case, $P_{x}=1$ and $P_{y}=1$, is the conventional discrete Hilbert transform for edge detection, and all directions of edges can be viewed in this case.

Example 4:

In this example, Lena image is used for our experiment. Fig.7 shows the edge detection results for various choices of parameters. The choices of parameters in this example are only for $P_{x}$ and $P_{y}$. The other two parameters $Q_{x}$ and $Q_{y}$ used in this example equal to 1 . It can be observed the edges in Lena image can be emphasized through different choices of parameters. The selections of threshold in this example are using the moment preserving method introduced in [8]. It can be observed that the edges can be detected through the choices for parameters $P_{x}$ and $P_{y}$.
The relations between selecting directions and parameters are the same as those of Example 3.

\section{CONCLUSION}

The discrete fractional Hilbert transform is proposed in this paper, and it has two ways to be defined the same as the continuous case. Similar to the continuous case, these two definitions can be combined into a general one. It is important that the proposed discrete fractional Hilbert transform has similar results as those of continuous fractional Hilbert transform. Moreover, the proposed discrete fractional Hilbert transform can be used in edge detections for digital images.

\section{REFERENCES}

[1] R. E. Ziemer and W. H. Tranter, Principles of Communications - Systems, Modulation, and Noise. Houghton Mifflin Company, 1990.

[2] K. Kohlmann, "Corner detection in natural images based on the 2-d Hilbert transform," Signal Processing, vol. 48, pp. 225-234, 1996.

[3] A. W. Lohmann, D. Mendlovic, and Z. Zalevsky, "Fractional Hilbert transform," Optics Letters, vol.21, pp. 281-283, Feb 1996.

[4] L. B. Jackson, Digital Filters and Signal Processing, Kluwer Academic Publisher, 1989.

[5] L. B. Almeida, "The fractional Fourier transform and time-frequency representation," IEEE Trans. Signal Process., vol. 42, pp. 3084-3091, Nov. 1994

[6] B. Santhanam and J. H. McClellan, "The discrete rotational Fourier transform," IEEE Trans. Signal Process., vol. 42, pp. 994-998, April 1996.

[7] S. C. Pei and M. H. Yeh, "Improved discrete fractional Fourier transform," Optics Letters, vol. 22, pp. 10471049, July 151997.

[8] W. Tsai "Moment preserving thresholding: A new approach," Computer Vision, Graphics, and Image Processing, vol. 29, pp. 377-393, 1985.

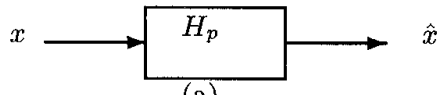

(a)

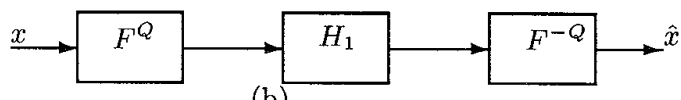

(b)

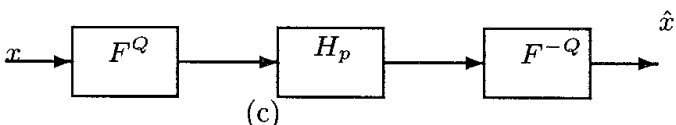

(c)

Figure 1: Block diagram for implementing fractional Hilbert Transform 


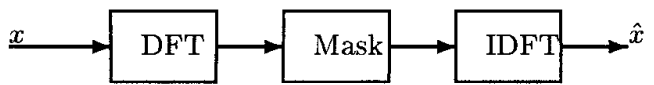

Figure 2: Implementation of Discrete Hilbert Transform
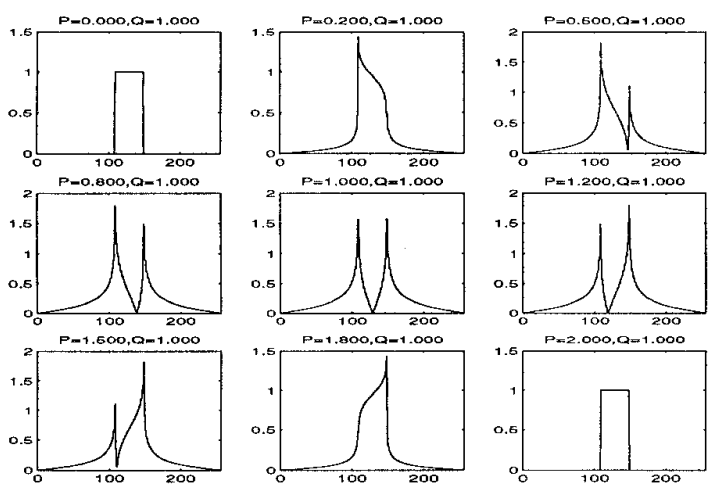

Figure 3: Discrete fractional Hilbert transform of a rectangular window
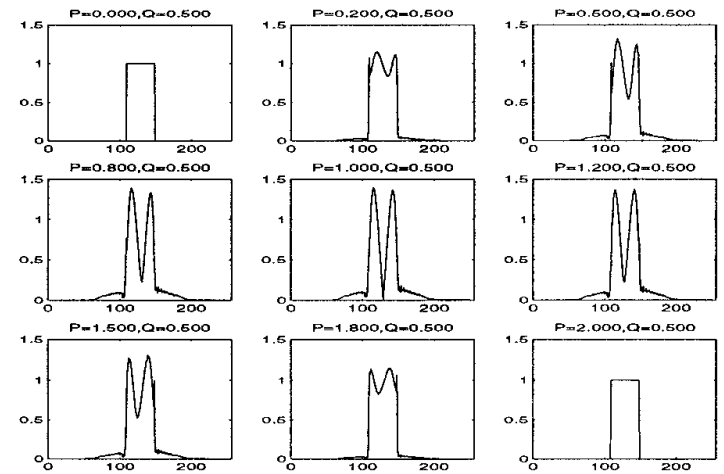

Figure 4: Discrete fractional Hilbert transform of a rectangular window
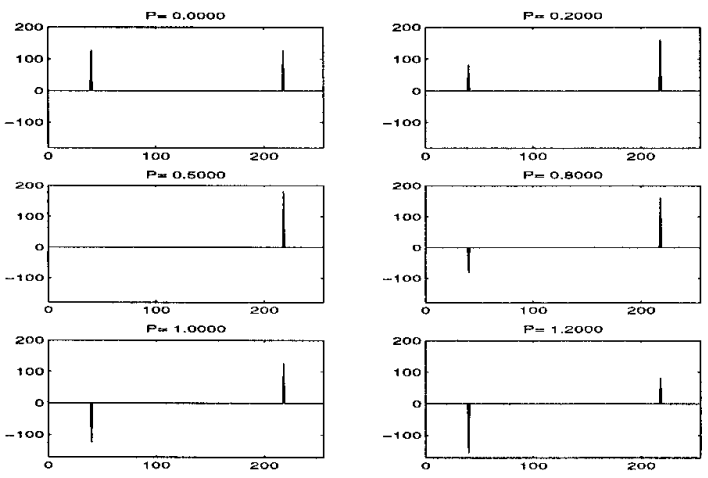

Figure 5: Fractional analytic signals of a sinusoids signal

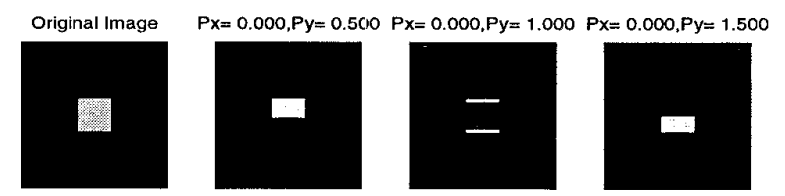

$P x=0.500, P y=0.000 P x=0.500, P y=0.5010 \quad P x=0.500, P y=1.000 \quad P x=0.500, P y=1.500$
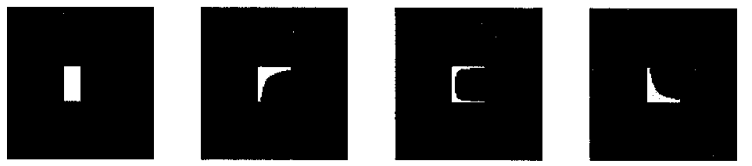

$P X=1.000, P y=0.000 \quad P X=1.000, P y=0.500 \quad P x=1.000, P y=1.000 \quad P X=1.000, P y=1.500$
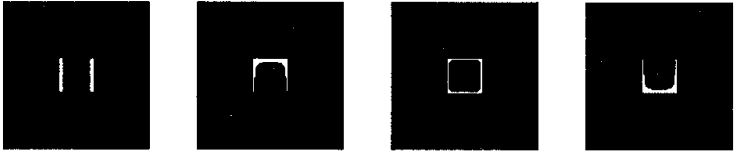

$P x=1.500, P y=0.000 \quad P x=1.500, P y=0.500 \quad P x=1.500, P y=1.000 \quad P x=1.500, P y=1.500$
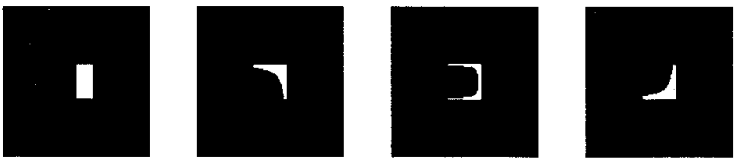

Figure 6: Results of Example 3

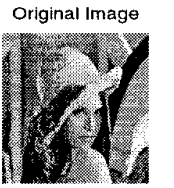

$P x=0.000, P y=0.5010 P x=0.000, P y=1.000 \quad P x=0.000, P y=1.500$

$P x=0.500, P y=0.000 P x=0.500, P y=0.5010 \quad P x=0.500, P y=1,000 \quad P x=0.500, P y=1.500$
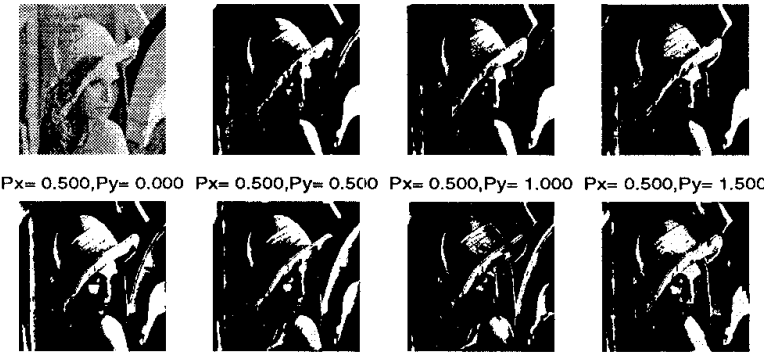

$P x=1.000, P y=0.000 \quad P x=1.000, P y=0.510 \quad P x=1.000, P y=1.000 \quad P x=1.000, P y=1.500$
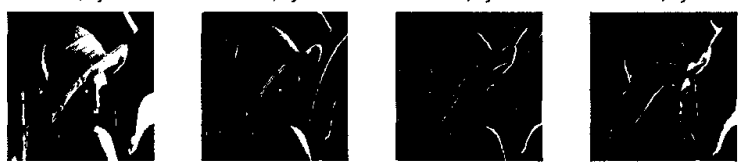

$P x=1500, P y=0.000 \quad P x=1.500, P y=0.500 \quad P x=1.500, P y=1.000 \quad P x=1.500, P y=1.500$
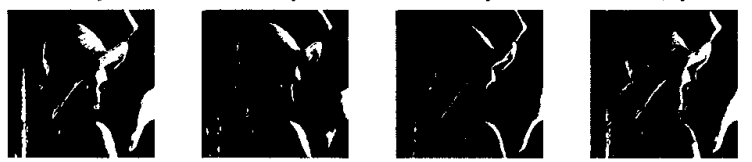

Figure 7: Results of Example 4 